\title{
Symbol-level precoding in MISO broadcast channels for SWIPT systems
}

\author{
Stelios Timotheou, Member, IEEE, Gan Zheng, Senior Member, IEEE, Christos Masouros, Senior Member, IEEE, \\ Ioannis Krikidis, Senior Member, IEEE
}

\begin{abstract}
This work investigates a problem for joint transmit beamforming and receive power splitting in multiple-input singleoutput downlink systems under quality of service and power transfer constraints. Rather than suppressing interference as in conventional schemes, this work takes advantage of constructive interference among users, inherent in the downlink, as a source of both useful information signal energy and electrical wireless energy. Specifically, we propose a new data-aided precoding design that minimizes the transmit power while guaranteeing the quality of service $(\mathrm{QoS})$ and energy harvesting constraints for generic phase shift keying modulated signals. The QoS constraints are modified to accommodate constructive interference, based on the constructive regions in the signal constellation.

Although the resulting problem is nonconvex, we propose second-order cone programming algorithms with polynomial complexity that provide upper and lower bounds to the optimal solution and establish the asymptotic optimality of these algorithms when the modulation order and signal to interferenceplus-noise ratio threshold tend to infinity. Simulation results show significant power savings with the proposed data-aided precoding approach compared to the conventional precoding scheme.
\end{abstract}

Keywords: SWIPT, Constructive interference, Beamforming, Power splitting, MISO channel, Optimization.

\section{INTRODUCTION}

Recently simultaneous wireless information and power transfer (SWIPT) via the radio frequency (RF) energy harvesting (EH) technology has emerged as a new solution for sustainable wireless network operation. In a multiuser scenario, interference signals provide a source for $\mathrm{EH}$, but at the same time may be harmful for QoS constraints.

The fundamental concept of SWIPT is introduced in [1] from an information theoretic standpoint, while [2] discusses two practical receiver SWIPT structures termed as "time switching" (TS) and "power splitting" (PS), that aim to optimally split the time and received signal power to achieve the QoS and EH constraints, respectively. In multi-antenna and multi-user systems, the optimal precoding design for SWIPT in a MISO broadcast channel is studied in [3], while the MISO interference channel (IC) case is studied in [4] based on PS receivers, using semidefinite programming (SDP). A more efficient and decentralized second-order cone programming (SOCP) relaxation is used in [5]. Multicell coordinated precoding has also been investigated in [6], in which Lagrangian optimization and semidefinite relaxation are used to solve the resulting nonconvex problem. In these works, conventional precoding design has been employed aiming to suppress interference, taking a statistical view of interference by focusing on either maximising the QoS - most commonly signal to interference-plus-noise ratio (SINR) - and minimizing interference subject to transmit power constraints, or minimizing the transmit power subject to QoS constraints.

A new branch of the downlink beamforming optimization literature, offers an alternative view of interference, where as opposed to the above statistical approach, interference is treated on an instantaneous basis, by symbol-level precoding. The relevant works focus on exploiting the constructive superposition of useful and interfering signals, to utilise interfering signals as first explored for closed-form precoders [7]- [8]. In [9] a symbol-level precoding is introduced where the conventional optimization constraints are adapted to accommodate constructive interference for phase shift keying modulation (PSK). Further work in [10] focuses on a more relaxed optimization where the optimization constraints are designed based on the constructive interference regions in the PSK constellation, first characterised in [11]. More recent work has extended the above downlink beamforming optimization to quadrature amplitude modulation (QAM) constellations [12].

In this work, we explore the adaptation of conventional SWIPT beamforming [1]- [4] as per the above symbol-level precoding, to exploit an interfering signal as both a source of useful electrical power for EH and information-driven signal power for the exploitation of constructive interference (CI) [13]. Our major contribution is that we introduce a new linear data-aided precoder design for SWIPT in the MISO broadcast channel with PS receivers using M-PSK modulation, that reduces the transmit power for given QoS and EH constraints compared to existing precoders. We simplify the original problem by re-casting it into a virtual multicast optimization program. Although the resulting problem is still non-convex, we develop upper bound (UB) and lower bound (LB) SOCP formulations that can be solved using standard optimization solvers. In addition, we prove asymptotic optimality of the developed LB and UB algorithms when the SINR threshold and the modulation order tends to infinity.

The rest of the paper is organized as follows. Section II introduces the system model and provides a review of conventional precoding design, while Section III formulates the considered optimization problem based on CI precoding. Section IV develops upper and lower bound solutions based on polynomial complexity SOCP formulations and studies the asymptotic performance of the developed algorithms. Section $\mathrm{V}$ illustrates the numerical performance of the developed algorithms compared to optimality and conventional precoding design. Finally, Section VI concludes the paper.

Notation: We use the upper case boldface letters for matrices and lower case boldface letters for vectors. $(\cdot)^{*}$ and $(\cdot)^{T}$ denote 
the conjugate and transpose, respectively. $\|\cdot\|$ stands for the Frobenius norm. A complex Gaussian random vector variable $\mathbf{z}$ with mean $\boldsymbol{\mu}$ and variance variance $\boldsymbol{\Sigma}$ is represented as $\mathbf{z} \sim$ $\mathcal{C N}(\boldsymbol{\mu}, \boldsymbol{\Sigma})$. A uniform random variable in the range $[a, b]$ is denoted by $z \sim U(a, b) . \mathbb{E}\{\cdot\}$ denotes the expectation. $\operatorname{Re}(x)$ and $\operatorname{Im}(x)$ denote the real part and imaginary part of a complex number $x \in \mathbb{C}$, respectively.

\section{System Model And Conventional PRecoding}

Consider a MISO broadcast channel where an $N$-antenna base station (BS) transmits both signals and energy to $K$ single-antenna users. For user $i$, its channel vector, precoding vector, received noise, data, SINR and EH constraints are denoted as $\mathbf{h}_{i}^{T}, \mathbf{t}_{i}, n_{i}, d_{i}, \Gamma_{i}, E_{i}$, respectively. The PSK modulated symbol can be expressed as $d_{i}=d e^{j \phi_{i}}$ where $d$ denotes the constant amplitude and $\phi_{i}$ is the phase, where for simplicity $d=1$. The average transmit power is $P_{T}=$ $\mathbb{E}\left\{\left\|\sum_{k=1}^{K} \mathbf{t}_{k} d_{k}\right\|^{2}\right\}$. The received signal at user $i$ is

$$
y_{i}=\mathbf{h}_{i}^{T} \sum_{k=1}^{K} \mathbf{t}_{k} d_{k}+n_{i}
$$

where $n_{i} \sim \mathcal{C} \mathcal{N}\left(0, N_{0}\right)$ is the additive white Gaussian noise (AWGN). To decode the information and harvest RF energy at the receiver side, the practical PS technique [2] is used. Specifically, the receiver splits the RF signal into two parts: one for information decoding and the other for energy harvesting, with relative power of $\rho_{i}$ and $1-\rho_{i}$, respectively.

The signal for information decoding is expressed as

$$
\tilde{y}_{i}=\sqrt{\rho_{i}} y_{i}+\tilde{n}_{i}=\sqrt{\rho_{i}} \mathbf{h}_{i}^{T} \sum_{k=1}^{K} \mathbf{t}_{k} d_{k}+\sqrt{\rho_{i}} n_{i}+\tilde{n}_{i},
$$

where $\tilde{n}_{i} \sim \mathcal{C N}\left(0, N_{C}\right)$ is the complex AWGN introduced in the RF to baseband conversion in the decoding process, which is independent of $n_{i}$.

The signal for energy harvesting is

$$
\bar{y}_{i}=\sqrt{1-\rho_{i}} y_{i}=\sqrt{1-\rho_{i}}\left(\mathbf{h}_{i}^{T} \sum_{k=1}^{K} \mathbf{t}_{k} d_{k}+n_{i}\right)
$$

with average power

$$
P_{i}=\left(1-\rho_{i}\right) \mathbb{E}\left\{\left|\mathbf{h}_{i}^{T} \sum_{k=1}^{K} \mathbf{t}_{k} d_{k}+n_{i}\right|^{2}\right\} .
$$

The problem of interest is to minimize the average transmit power $P_{T}$ subject to QoS (i.e., SINR) constraints $\left\{\Gamma_{i}\right\}$ and energy harvesting constraints $\left\{E_{i}\right\}$, respectively. This will be achieved by optimizing beamforming design, power allocation and splitting, by exploiting the CI concept.

In conventional MISO downlink precoding, users' data are independent of each other, i.e., $\mathbb{E}\left(d_{i}^{*} d_{j}\right)=0, \forall j \neq i$. In this case, the transmit power in becomes $P_{T}=\sum_{i=1}^{K}\left\|\mathbf{t}_{i}\right\|^{2}$.

Based on the signal model (2) for information decoding, the received SINR for user $i$ is given by

$$
\Gamma_{i}^{c o n}=\frac{\left|\mathbf{h}_{i}^{T} \mathbf{t}_{i}\right|^{2}}{\sum_{j=1, j \neq i}^{K}\left|\mathbf{h}_{i}^{T} \mathbf{t}_{j}\right|^{2}+N_{0}+\frac{N_{C}}{\rho_{i}}} .
$$

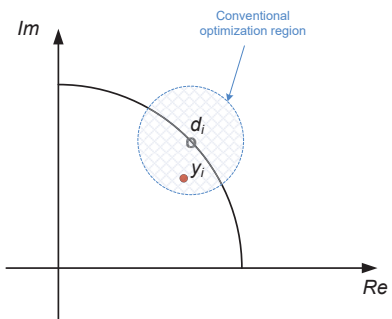

(a)

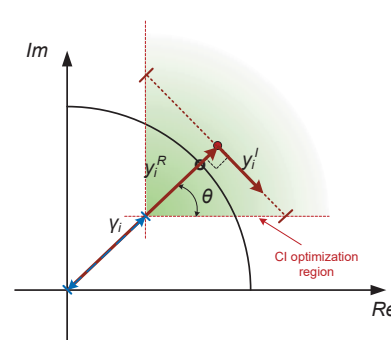

(b)
Fig. 1. Illustration of constructive interference for information decoding, QPSK example.

The harvested energy is equal to

$$
P_{i}^{c o n}=\left(1-\rho_{i}\right)\left(\sum_{k=1}^{K}\left|\mathbf{h}_{i}^{T} \mathbf{t}_{k}\right|^{2}+N_{0}\right) .
$$

Consequently, the power minimization problem with both QoS and $\mathrm{EH}$ constraints can be formulated as

$$
\begin{aligned}
\min _{\left\{\mathbf{t}_{i}, \rho_{i}\right\}} & \sum_{i=1}^{K}\left\|\mathbf{t}_{i}\right\|^{2} \\
\text { s.t. } & \Gamma_{i}^{c o n} \geq \Gamma_{i}, P_{i}^{c o n} \geq E_{i}, 0<\rho_{i}<1, \forall i .
\end{aligned}
$$

It is easy to see that formulation (6), which we call SDPnoCI, is non-convex and hence challenging to solve. Fortunately, the authors of [3] have shown that the SDP relaxation of SDPnoCI is tight for the general MISO downlink case.

\section{DATA-Aided PRECoding Problem}

With the knowledge of both the instantaneous CSI and the data symbols at the BS, the received interference can be classified to be constructive or destructive. In brief, while destructive interference deteriorates performance, CI moves the received symbols away from the decision thresholds of the constellation and thus improves the detection. We refer the readers to [7], [8] for further details. The main idea of the proposed precoding is to exploit the CI for both information decoding and energy harvesting.

The received signal at user $i$ in (1) can be rewritten as

$$
y_{i}=\mathbf{h}_{i}^{T} \sum_{k=1}^{K} \mathbf{t}_{k} d_{k}+n_{i}=\mathbf{h}_{i}^{T} \sum_{k=1}^{K} \mathbf{t}_{k} e^{j\left(\phi_{k}-\phi_{i}\right)} d_{i}+n_{i} .
$$

The information decoding part can be written as

$\tilde{y}_{i}=\sqrt{\rho_{i}} y_{i}+\tilde{n}_{i}=\sqrt{\rho_{i}} \mathbf{h}_{i}^{T} \sum_{k=1}^{K} \mathbf{t}_{k} e^{j\left(\phi_{k}-\phi_{i}\right)} d_{i}+\sqrt{\rho_{i}} n_{i}+\tilde{n}_{i}$.

We illustrate the derivation of the SINR constraint for the example of QPSK in Fig. 1. The reader is referred to [10] for further details where this concept is explained in the context of downlink precoding. Here, Fig. 1(a) represents the conventional optimization region and Fig. 1(b) shows the proposed optimization region. We have used the definitions $y_{i}^{R}=\operatorname{Re}\left(\mathbf{h}_{i}^{T} \sum_{k=1}^{K} \mathbf{t}_{k} e^{j\left(\phi_{k}-\phi_{i}\right)}\right), y_{i}^{I}=$ $\operatorname{Im}\left(\mathbf{h}_{i}^{T} \sum_{k=1}^{K} \mathbf{t}_{k} e^{j\left(\phi_{k}-\phi_{i}\right)}\right)$ and $\gamma_{i}=\sqrt{\Gamma_{i}\left(N_{0}+\frac{N_{C}}{\rho_{i}}\right)}$. 
In conventional precoding optimization, $y_{i}^{R}$ and $y_{i}^{I}$ are constrained such that the received symbol is contained within a circle (denoted by the dashed circle in Fig. 1(a)) around the nominal constellation point, so that the interference caused by the other symbols is limited. In contrast to this, the concept of CI is exploited to allow a relaxation of $y_{i}^{R}$ and $y_{i}^{I}$ for all transmit symbols, under the condition that the interference caused is constructive, lying in the green shaded sector in the diagram [8]. It can be seen that $y_{i}^{R}$ and $y_{i}^{I}$ are allowed to grow infinitely, as long as their ratio is kept such that the received symbol is contained within the constructive area of the constellation, i.e., the distances from the decision thresholds, as set by the SNR constraints $\gamma_{i}$, are not violated. It can be seen that the angle of interference need not be strictly aligned with the angle of the useful signal, as long as it falls within the constructive area of the constellation. For a given modulation order $M$ the maximum angle shift in the CI area is given by $\theta=\pi / M$. By using basic geometry we arrive at the SINR constraint expressed as [10]

which is expanded to

$$
\left|y_{i}^{I}\right| \leq\left(y_{i}^{R}-\gamma_{i}\right) \tan \theta
$$

$$
\begin{aligned}
& \left|\operatorname{Im}\left(\mathbf{h}_{i}^{T} \sum_{k=1}^{K} \mathbf{t}_{k} e^{j\left(\phi_{k}-\phi_{i}\right)}\right)\right| \leq \\
& \left(\operatorname{Re}\left(\mathbf{h}_{i}^{T} \sum_{k=1}^{K} \mathbf{t}_{k} e^{j\left(\phi_{k}-\phi_{i}\right)}\right)-\sqrt{\Gamma_{i}\left(N_{0}+\frac{N_{C}}{\rho_{i}}\right)}\right) \tan \theta .
\end{aligned}
$$

The harvested energy and the total transmit power are given by $P_{i}=\left|\mathbf{h}_{i}^{T} \sum_{k=1}^{K} \mathbf{t}_{k} e^{j\left(\phi_{k}-\phi_{i}\right)}\right|^{2}$ and $P_{T}=$ $\left\|\sum_{k=1}^{K} \mathbf{t}_{k} e^{j\left(\phi_{k}-\phi_{i}\right)}\right\|^{2}$, respectively. By defining $\tilde{\mathbf{h}}_{i}=$ $\mathbf{h}_{i} e^{j\left(\phi_{1}-\phi_{i}\right)}$ and $\mathbf{w} \triangleq \sum_{k=1}^{K} \mathbf{t}_{k} e^{j\left(\phi_{k}-\phi_{1}\right)}$, the power minimization problem subject to both SINR and EH constraints with the aid of the CI can be formulated as

$$
\begin{aligned}
& \min _{\{\mathbf{w}, \boldsymbol{\rho}\}}\|\mathbf{w}\|^{2} \\
& \text { s.t. }\left|\operatorname{Im}\left(\tilde{\mathbf{h}}_{i}^{T} \mathbf{w}\right)\right| \leq\left(\operatorname{Re}\left(\tilde{\mathbf{h}}_{i}^{T} \mathbf{w}\right)\right. \\
& \left.\quad-\sqrt{\Gamma_{i}\left(N_{0}+\frac{N_{C}}{\rho_{i}}\right)}\right) \tan \theta, i \in \mathcal{K} \\
& \quad\left\|\tilde{\mathbf{h}}_{i}^{T} \mathbf{w}\right\|^{2} \geq \frac{E_{i}}{1-\rho_{i}}, i \in \mathcal{K} \\
& 0<\rho_{i}<1, i \in \mathcal{K} .
\end{aligned}
$$

Although the reformulation (11) seems a trivial step, it indicates that the original broadcast channel reduces to a virtual multicast channel with common messages to all users [14]. The problem (11) is challenging to solve because of the nonconvex constraint $\left|\tilde{\mathbf{h}}_{i}^{T} \mathbf{w}\right|^{2} \geq \frac{E_{i}}{1-\rho_{i}}$. The rest of this paper is devoted to solving the multicast problem (11).

\section{Polynomial COMPLEXITy SOLUTIONS}

Here, we develop an upper and lower bounding algorithm for (11) using convex SOCP.

\section{A. SOCP-UB: Upper bounding SOCP algorithm}

In this section, we develop SOCP-UB, an upper bound solution to (11) derived by convexifying the problem using SOCP. Towards this direction, we begin by reformulating (11b) for $i \in \mathcal{K}$ using SOCP constraints. If we define

$$
\begin{aligned}
v_{i} & =\left|\operatorname{Im}\left(\tilde{\mathbf{h}}_{i}^{T} \mathbf{w}\right)\right|, \\
y_{i}^{R} & =\operatorname{Re}\left(\tilde{\mathbf{h}}_{i}^{T} \mathbf{w}\right)=\sum_{k=1}^{K} \operatorname{Re}\left(\tilde{h}_{i, k}\right) w_{k}^{R}-\operatorname{Im}\left(\tilde{h}_{i, k}\right) w_{k}^{I}, \\
y_{i}^{I} & =\operatorname{Im}\left(\tilde{\mathbf{h}}_{i}^{T} \mathbf{w}\right)=\sum_{k=1}^{K} \operatorname{Im}\left(\tilde{h}_{i, k}\right) w_{k}^{R}+\operatorname{Re}\left(\tilde{h}_{i, k}\right) w_{k}^{I},
\end{aligned}
$$

then it is true that the absolute term of (11b) can be equivalently represented by two linear constraints as:

$$
y_{i}^{I} \leq v_{i}, \quad-y_{i}^{I} \leq v_{i}, i \in \mathcal{K} .
$$

This is true because on the one hand, constraint (14) forces $v_{i} \geq\left|y_{i}^{I}\right|, i \in \mathcal{K}$, and on the other hand, (11b) forces $v_{i}$ to be as small as possible, which is achieved for $v_{i}=\left|y_{i}^{I}\right|$.

To deal with the square root, the terms in (11b) are rearranged and both sides of the constraint are squared yielding $\left(y_{i}^{R}-v_{i} / \tan \theta\right)^{2} \geq \Gamma_{i}\left(N_{0}+\frac{N_{C}}{\rho_{i}}\right)$ which is equivalent to

$$
\begin{aligned}
z_{i}^{+} & =y_{i}^{R}-v_{i} / \tan \theta+\sqrt{\Gamma_{i} N_{0}}, \\
z_{i}^{-} & =y_{i}^{R}-v_{i} / \tan \theta-\sqrt{\Gamma_{i} N_{0}}, \\
z_{i}^{+} z_{i}^{-} & \geq \frac{\Gamma_{i} N_{C}}{\rho_{i}} .
\end{aligned}
$$

From the constraint in (11b), it is easy to see that a solution of (11) satisfies $y_{i}^{R}>v_{i} / \tan \theta \geq 0$, and hence $z_{i}^{+}>0$. Because the right hand side (r.h.s.) of (17) is positive, combined with the fact that $z_{i}^{+}>0$, implies that a solution of (11) satisfies $z_{i}^{+}, z_{i}^{-}>0$;

$$
\begin{aligned}
& z_{i}^{+} z_{i}^{-} \geq r_{1, i}^{2}, \quad \rho_{i} \geq r_{2, i}^{2}, \\
& r_{1, i} r_{2, i} \geq \sqrt{\Gamma_{i} N_{C}}, \\
& z_{i}^{+} \geq 0, z_{i}^{-} \geq 0, r_{1, i} \geq 0, r_{2, i} \geq 0 .
\end{aligned}
$$

In sum, constraint (11b) is expressed by (12)-(16) and (18)(20).

Constraint (11c), is not convex due to the term $\left\|\tilde{\mathbf{h}}_{i}^{T} \mathbf{w}\right\|^{2}=$ $\left(y_{i}^{R}\right)^{2}+\left(y_{i}^{I}\right)^{2}, i \in \mathcal{K}$; nonetheless, it can be convexified by eliminating the real or imaginary part. Eliminating the imaginary part is better because $y_{i}^{R} \geq\left|v_{i}\right| / \tan \theta+\sqrt{\Gamma_{i} N_{0}}$ yielding the constraint:

$$
\left(y_{i}^{R}\right)^{2} \geq E_{i} /\left(1-\rho_{i}\right),
$$

which is similar to (17), and can be reformulated into SOCP constraints. Hence, the approximate SOCP formulation is:

$$
\min _{\left\{\mathbf{w}, \boldsymbol{\rho}, \mathbf{z}_{i}^{ \pm}, \mathbf{r}_{1}, \mathbf{r}_{2}, \mathbf{r}_{3}, \mathbf{y}^{R}, \mathbf{y}^{I}, \mathbf{v}\right\}}\|\mathbf{w}\|^{2}
$$

s.t. Constraints (11d), (12)-(16), (18)-(20),

$1-\rho_{i} \geq r_{3, i}^{2}, i \in \mathcal{K}$,

$r_{3, i} y_{i}^{R} \geq \sqrt{E_{i}}, i \in \mathcal{K}$,

$r_{3, i} \geq 0, y_{i}^{R} \geq 0 i \in \mathcal{K}$. 
Problem (22) provides an upper bound to the solution of (11), as its solution is always feasible for the latter since $\left(y_{i}^{R}\right)^{2}+\left(y_{i}^{I}\right)^{2} \geq\left(y_{i}^{R}\right)^{2}$. Note that if $y_{i}^{I}=0, i \in \mathcal{K}$, then this formulation provides an optimal solution.

\section{B. SOCP-LB: SOCP lower bounding algorithm}

In order to obtain an SOCP LB solution, we need to approximate from below the non-convex term in (11c). For this reason, we consider that $y_{i}^{R} \geq v_{i} / \tan \theta$, which implies that $\left(y_{i}^{R}\right)^{2}+y_{i}^{R} v_{i} \tan \theta \leq\left(y_{i}^{R}\right)^{2}+\left(y_{i}^{I}\right)^{2}$. Because $\left(y_{i}^{R}\right)^{2}+y_{i}^{R} v_{i} \tan \theta$ can be expressed as the product of two positive linear terms, i.e., $y_{i}^{R}\left(y_{i}^{R}+v_{i} \tan \theta\right)$, the resulting constraint can be expressed into a convex SOCP form similar to (17), yielding the formulation:

$$
\min _{\left\{\mathbf{w}, \boldsymbol{\rho}, \mathbf{z}_{i}^{ \pm}, \mathbf{r}_{1}, \mathbf{r}_{2}, \mathbf{r}_{3}, \mathbf{r}_{4}, \mathbf{u}, \mathbf{y}^{R}, \mathbf{y}^{I}, \mathbf{v}\right\}}\|\mathbf{w}\|^{2}
$$

s.t. Constraints (11d), (12)-(16), (18)-(20),(22c),

$$
\begin{aligned}
& u_{i}=y_{i}^{R}+v_{i} \tan \theta, i \in \mathcal{K}, \\
& y_{i}^{R} u_{i} \geq r_{4, i}^{2}, i \in \mathcal{K}, \\
& r_{3, i} r_{4, i} \geq \sqrt{E_{i}}, i \in \mathcal{K}, \\
& r_{3, i} \geq 0, r_{4, i} \geq 0, u_{i} \geq 0, i \in \mathcal{K} .
\end{aligned}
$$

\section{Asymptotic optimallity}

In this section we prove that the developed algorithms asymptotically converge to the optimal solution when the SINR threshold $\left(\Gamma_{i}\right)$ and the modulation order $(M)$ tends to infinity. The main result is as follows:

Proposition 1. The solutions of (22) and (23) asymptotically converge to the optimum for $M \rightarrow \infty$ and for $\Gamma_{i} \rightarrow \infty, i \in \mathcal{K}$.

Proof: The proof is provided in Appendix A.

Proposition 1 is important because practical systems often use a large modulation order and high SINR threshold to achieve high data rates and low outage probability, in which cases the proposed algorithms will achieve asymptotically optimal results. The simulation section verifies this result for practical communication scenarios.

\section{Simulation Results}

In this section we investigate the benefits of using CI, examine the performance of the developed algorithms and experimentally verify the theoretical findings. The considered setting involves $K$ receivers randomly located around the BS with distance $l_{i}$ and direction $\zeta_{i}$ drawn from the uniform distribution, $l_{i} \sim U(2,7) \mathrm{m}$ and $\zeta_{i} \sim U(-\pi, \pi)$. Each receiver can harvest energy at frequency $f=915 \mathrm{MHz}$ while it is assumed that the gains per antenna at the BS and receivers are $8 \mathrm{dBi}$ and $3 \mathrm{dBi}$, respectively. The path attenuation of receiver $i$, $L_{i}$, is obtained using the Friis equation with reference distance $1 \mathrm{~m}$ and path loss coefficient 2.5. It is further assumed that $K=N=4, N_{0}=-70 \mathrm{dBm}$ and $N_{C}=-50 \mathrm{dBm}$, while the EH and SINR thresholds are the same for all receivers, i.e. $\Gamma_{i}=\Gamma, E_{i}=E, i \in \mathcal{K}$. The modulation scheme used is QPSK unless otherwise stated. Rician fading is used to model the channel as the short distance between the BS and the receivers implies dominance of the line-of-sight (LOS) signal.
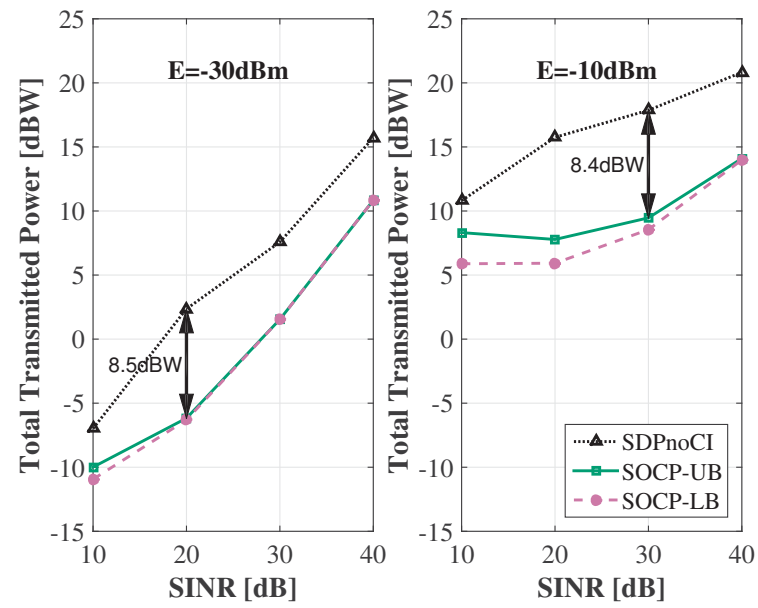

Fig. 2. Total transmitted power of investigated algorithms for varying $\Gamma$ when $E=\{-30,-10\} \mathrm{dBm}$ and $K=4$.
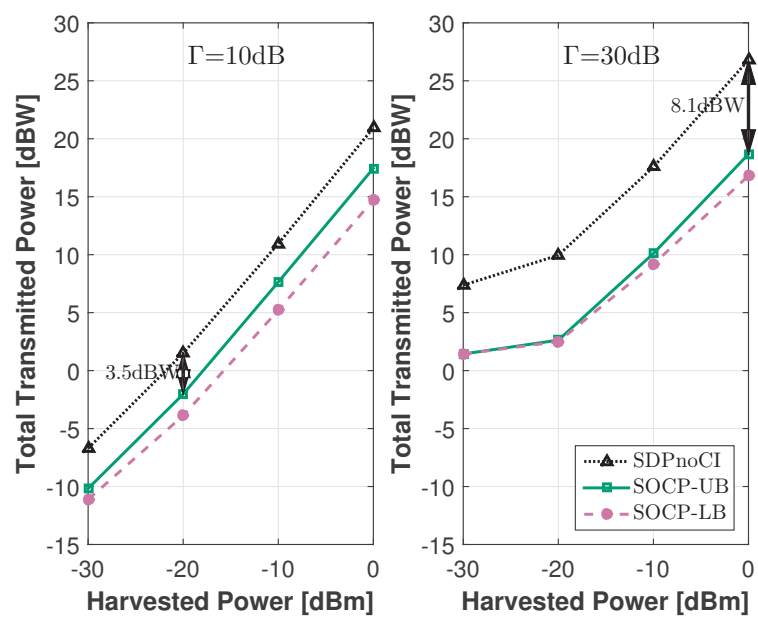

Fig. 3. Total transmitted power of investigated algorithms for varying $E$ when $\Gamma=\{10,30\} \mathrm{dB}$ and $K=4$.

Hence, $\mathbf{h}_{i}$ is composed of the LOS signal, $\mathbf{h}_{i}^{L O S}$ and the nonLOS signal $\mathbf{h}_{i}^{N L O S}$ according to the expression [3]

$$
\mathbf{h}_{i}=\sqrt{\frac{K_{R}}{1+K_{R}}} \mathbf{h}_{i}^{L O S}+\sqrt{\frac{1}{1+K_{R}}} \mathbf{h}_{i}^{N L O S},
$$

where $K_{R}=5 \mathrm{~dB}$ is the Rician factor. For the LOS signal the far-field uniform linear antenna array model with $\lambda / 2$ distance between antenna elements is considered [15] which implies that $\mathbf{h}_{i}^{L O S}=\sqrt{L_{i}}\left[1, \mathrm{e}^{-j\left(1 \pi \sin \zeta_{i}\right)}, \ldots, \mathrm{e}^{-j\left((N-1) \pi \sin \zeta_{i}\right)}\right]^{T}$. Rayleigh fading is adopted for the NLOS signal, $\mathbf{h}_{i}^{N L O S} \in$ $\mathbb{C}^{N \times 1}$ which means that each of its elements are circularly symmetric complex Gaussian (CSCG) random variables with zero mean and variance $L_{i}$.

We compare the performance of algorithms SDPnoCI, SOCP-UB and SOCP-LB based on (6), (22), and (23), respectively. Note that all figures depict results averaged over 1000 randomly generated problem instances per scenario.

Figs. 2, and 3 depict the total transmitted power achieved by the different investigated algorithms with varying $\Gamma$ and $E$, respectively. One important observation is that the per- 


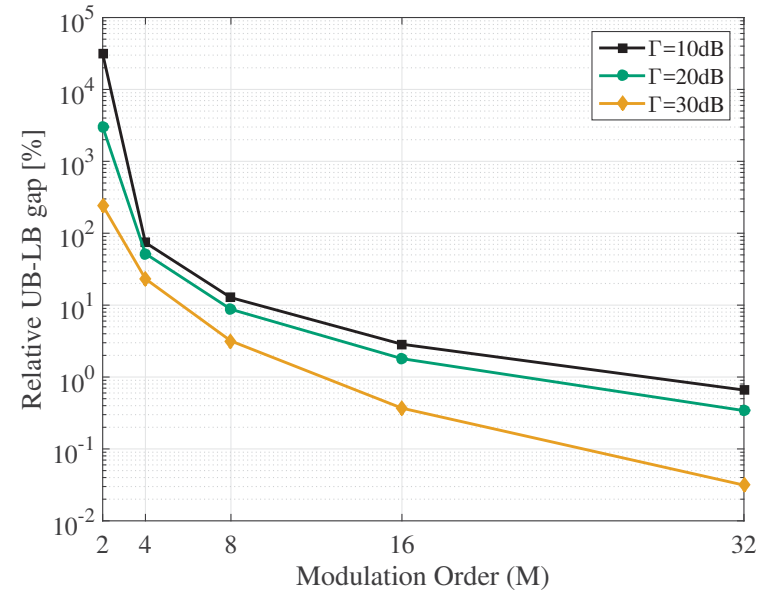

Fig. 4. Relative percentage gap between the solution of SOCP-UB and SOCPLB varying $\Gamma$ when $E=-10 \mathrm{dBm}$ and $K=8$.

formance of the conventional precoding scheme SDPnoCI is significantly worse than SOCP-UB. In fact, for varying $\Gamma$ and $E$ there is a performance gap between SOCP-UB and SDPnoCI in the range $[2.5,8.5] \mathrm{dBW}$ and $[3.5,8.1] \mathrm{dBW}$, respectively. In addition, Fig. 2 indicates that while SINR increases, the gap between SOCP-UB and SOCP-LB tends to zero, as expected from Proposition 1.

To demonstrate that the same is true for the modulation order $M$, we depict in Fig. 4 the relative percentage gap between the SOCP-UB and SOCP-LB as the modulation order increases from $M=2$ (BPSK) to $M=32$ (32-PSK). It can be observed that the optimality gap reduces by four orders of magnitude as $M$ is increased, achieving an optimality gap smaller than $1 \%$ for $M=32$.

\section{CONCLUSIONS}

In this paper, we have explored the exploitation of the constructive interference in MISO downlink to boost the performance for both information decoding and energy harvesting. We have shown that, by means of data-aided beamforming, constructive interference can be exploited to improve the signal power as well as act as a source of wireless power transfer. Despite the fact that the formulated problem is nonconvex, lower and upper bound polynomial complexity solutions have been developed that provide results close to optimality, as well as reduced transmission power by 3-8 $\mathrm{dBW}$ compared to conventional precoding design. In addition, it has been theoretically proven that the proposed algorithms asymptotically converge to the optimal solution when the SINR threshold and the modulation order tend to infinity.

\section{APPENDIX A:Proof OF PROPOSITION 1}

First we prove asymptotic optimality for the modulation order. Since the solution of (22) provides an UB and the solution of (23) a LB, it suffices to show that as $M \rightarrow \infty$, the two formulations become identical which means that the gap between a feasible solution and a lower bound solution is equal to zero. Observing the approximated $\mathrm{EH}$ terms from formulations (22) and (23) is can be easily deduced that

$$
\left(y_{i}^{R}\right)^{2} \leq\left(y_{i}^{R}\right)^{2}+\left(y_{i}^{I}\right)^{2} \leq y_{i}^{R}\left(y_{i}^{R}+\left|y_{i}^{I}\right| \tan \theta\right)
$$

For $M \rightarrow \infty$ it is true that $\tan \theta \rightarrow 0$ which means that $\left(y_{i}^{R}\right)\left(y_{i}^{R}+\left|y_{i}^{I}\right| \tan \theta\right) \rightarrow\left(y_{i}^{R}\right)^{2}$, and based on (25) this also implies that $\left|y_{i}^{I}\right| \rightarrow 0$. This is also verified from the SINR constraint as for $\tan \theta \rightarrow 0$ it must be true that $\left|y_{i}^{I}\right| \leq \mu, \mu \rightarrow 0$, which holds true when $\left|y_{i}^{I}\right| \rightarrow 0$. Hence, we have proven that for $M \rightarrow \infty$ the UB and LB EH constraints tend to the accurate $\mathrm{EH}$ constraint, completing the proof for the modulation order.

Regarding asymptotic optimality for $\Gamma_{i} \rightarrow \infty, i \in \mathcal{K}$, it is true from (16) that $y_{i}^{R} \geq\left|y_{i}^{I}\right| / \tan \theta+\sqrt{\Gamma_{i}\left(N_{0}+N_{C}\right)}$; hence, it is true that $y_{i}^{R}+\left|y_{i}^{I}\right| \tan \theta \rightarrow y_{i}^{R}$ so that $y_{i}^{R}\left(y_{i}^{R}+\right.$ $\left.\left|y_{i}^{I}\right| \tan \theta\right) \rightarrow\left(y_{i}^{R}\right)^{2}$. This shows that for $\Gamma_{i} \rightarrow \infty$ the upper and lower bound formulations ((22) and (23)) provide identical solutions which implies optimality, completing the proof.

\section{ACKNOWLEDGEMENT}

This work was supported by the Royal Academy of Engineering, UK and the Engineering and Physical Sciences Research Council (EPSRC) project EP/M014150/1.

\section{REFERENCES}

[1] P. Grover and A. Sahai, "Shannon meets Tesla: wireless information and power transfer," in Proc. IEEE Int. Symp. Inf. Theory, Austin, USA, June 2010, pp. 2363-2367.

[2] R. Zhang and C. K. Ho, "MIMO broadcasting for simultaneous wireless information and power transfer," IEEE Trans. Wireless Commun., vol. 12, no. 5, pp. 1989-2001, May 2013.

[3] Q. Shi, L. Liu, W. Xu, and R. Zhang, "Joint transmit beamforming and receive power splitting for MISO SWIPT systems," IEEE Trans. Wireless Commun., vol, 13, no. 6, pp. 3269-3280, June 2014.

[4] S. Timotheou, I. Krikidis, G. Zheng, and B. Ottersten, "Beamforming for MISO interference channels with QoS and RF energy transfer," IEEE Trans. Wireless Commun., vol. 13, no. 5, pp. 2646-2658, May 2014.

[5] Q. Shi, W. Xu, T-H Chang, Y. Wang and E. Song, "Joint Beamforming and Power Splitting for MISO Interference Channel With SWIPT: An SOCP Relaxation and Decentralized Algorithm," IEEE Trans. Signal Processing, vol.62, pp. 6149-6208, 2014.

[6] S. He, Y. Huang, W. Chen, S. Jin, H. Wang, and L. Yang, "Energy Efficient Coordinated Precoding Design for Multi-cell System with RF Energy Harvesting," EURASIP Journal on Wireless Communications and Networking, vol 67, pp.1-12, 2015.

[7] C. Masouros, and E. Alsusa, "Dynamic linear precoding for the exploitation of known interference in MIMO broadcast systems," IEEE Trans. Wireless Commun., vol. 8, no. 3, pp. 1396-1404, Mar. 2009.

[8] C. Masouros, "Correlation rotation linear precoding for MIMO broadcast communications", IEEE Trans. Signal Process., vol. 59, no. 1, pp. 252 262, Jan. 2011

[9] M. Alodeh, S. Chatzinotas and B. Ottersten, "Constructive Multiuser Interference in Symbol Level Precoding for the MISO Downlink Channel,” IEEE Trans. Signal Process., vol. 63 , no. 9 , pp. 2239-2252, May 2015.

[10] C. Masouros and G. Zheng, "Exploiting Known Interference as Green Signal Power for Downlink Beamforming Optimization," IEEE Trans. Signal Process., vol.63, no.14, pp.3668-3680, July, 2015.

[11] C. Masouros and E. Alsusa, "Soft Linear Precoding for the Downlink of DS/CDMA Communication Systems," IEEE Trans. on Veh. Tech., vol. 59, no. 1, pp. 203-215, Jan 2010

[12] M. Alodeh, S. Chatzinotas, and B. Ottersten, "Symbol-Level Multiuser MISO Precoding for Multi-level Adaptive Modulation: A Multicast View," available on Arxiv in 12th January 2016.

[13] G. Zheng, I. Krikidis, C. Masouros, S. Timotheou, D. A. Toumpakaris, and Z. Ding, "Rethinking the Role of Interference in Wireless Networks," IEEE Commun. Mag., vol.52, no.11, pp.152,158, Nov. 2014.

[14] N. D. Sidiropoulos, T. N. Davidson, and Z. Q. Luo, "Transmit beamforming for physical-layer multicasting," IEEE Trans. Signal Proc., vol. 54, no. 6, pp. 2239-2251, June 2006.

[15] E. Karipidis, N. D. Sidiropoulos, and Z. Q. Luo, "Far-field multicast beamforming for uniform linear antenna arrays," IEEE Trans. Signal Process., vol. 55, no. 10, pp. 4916-4927, 2007. 